(C)2009 IEEE. Personal use of this material is permitted. However, permission to reprint/republish this material for advertising or promotional purposes or for creating new collective works for resale or redistribution to servers or lists, or to reuse any copyrighted component of this work in other works must be obtained from the IEEE. 


\title{
Achieving a nonovershooting transient response with multivariable dynamic output feedback tracking controllers
}

\author{
Robert Schmid and Lorenzo Ntogramatzidis
}

\begin{abstract}
We consider the use of dynamic output feedback control to improve the transient response to a step input, for invertible multivariable systems. A method is given for designing a linear time-invariant output feedback controller to asymptotically track a constant step reference with zero overshoot and arbitrarily small rise time, under some mild assumptions.
\end{abstract}

\section{INTRODUCTION}

The problem of designing control laws to ensure a linear time invariant (LTI) plant has desirable transient response to a step input has been studied for the past few decades. The primary performance objectives include achieving a small rise time while also minimising overshoot.

Some recent papers have considered the problem of designing a suitable closed-loop feedback controller to achieve a non-overshooting response. For continuous time singleinput single-output (SISO) systems, in [1] an eigenstructure assignment method is given to obtain a non-overshooting LTI state feedback controller for plants with one non-minimum phase zero. Stable non-minimum phase SISO systems are considered in [2], where the existence of an output feedback controller is proved to give a non-overshooting step response, provided the plant has no zeros on the imaginary axis. In [3] it is shown how to give two parameter feedback controller for an LTI plant that renders the step response nonovershooting. A common feature of these recent papers [1][3] was that they considered only SISO systems, which were assumed to be initially at rest. In some cases the transient response was deliberately slowed to avoid overshoot, which necessarily yielded a lengthy settling time. In [4], the authors used a composite nonlinear (CNF) state-feedback controller consisting of a linear term chosen to yield a rapid response, and a nonlinear component designed to reduce overshoot. A smooth nonlinear error function is employed to tune the closed-loop dynamics. The authors showed their state feedback controller could also be implemented in conjunction with an observer scheme. However, their CNF scheme did not guarantee the step response will be non-overshooting.

In our recent paper [6], the present authors considered invertible stabilisable MIMO systems, and used linear statefeedback control to design a non-overshooting controller for a step reference. The design methods proposed there make use of the combined eigenvalue and eigenvector placement

R. Schmid is with the Department of Electrical and Electronic Engineering, The University of Melbourne, Parkville, Vic. 3010, Australia. E-mail: rschmideunimelb. edu. au

L. Ntogramatzidis is with the Department of Mathematics and Statistics, Curtin University of Technology, Perth, WA, Australia. E-mail: L.Ntogramatzidisecurtin.edu.au methods for state feedback given in [5] by B.C. Moore, and are applicable to both continuous time and discrete time systems. Moreover, the design method is applicable to both minimum phase and non-minimum phase systems. Conditions are given under which a linear state-feedback controller can be obtained to asymptotically track a step reference with guaranteed zero overshoot, from any initial condition. The controller can be readily chosen to achieve any desired convergence rate.

In this paper we continue our investigation of the tracking problem considered in [6], and consider the implementation and performance of the design methods given in those papers when implemented with dynamic output feedback based on a Luenberger observer scheme. We will show that state feedback tracking control scheme given in those papers can also be successfully implemented with a dynamic output feedback scheme, provided the initial state estimation error is sufficiently small.

\section{Problem Formulation}

We consider the LTI system $\Sigma$ characterized by

$$
\Sigma:\left\{\begin{array}{l}
\dot{x}(t)=A x(t)+B u(t), x(0)=x_{0}, \\
y(t)=C x(t)+D u(t),
\end{array}\right.
$$

where, for all $t \in \mathbb{R}, x(t) \in \mathbb{R}^{n}$ is the state, $u(t) \in \mathbb{R}^{m}$ is the control input, $y(t) \in \mathbb{R}^{p}$ is the output, and $A, B, C$ and $D$ are appropriate dimensional constant matrices.

In this paper we are concerned with the problem of designing an output feedback control law for (1) such that the output $y$ of (1) tracks a step reference $r \in \mathbb{R}^{p}$ with zero steady-state error with no overshoot. As such, we make the following standing assumption:

Assumption 2.1: The system $\Sigma$ is invertible, stabilizable, detectable and has no invariant zeros at the origin.

The following method for designing a tracking controller for a constant step reference $r \in \mathbb{R}^{p}$ is standard: By virtue of Assumption 2.1, we may obtain gain matrices $F$ and $G$ such that $A+B F$ and $A+G C$ are both stable matrices, and there exist vectors $x_{\mathrm{ss}} \in \mathbb{R}^{n}$ and $u_{\mathrm{ss}} \in \mathbb{R}^{m}$ exist satisfying

$$
\begin{aligned}
& 0=A x_{\mathrm{ss}}+B u_{\mathrm{ss}} \\
& r=C x_{\mathrm{ss}}+D u_{\mathrm{ss}}
\end{aligned}
$$

for any $r \in \mathbb{R}^{p}$. We introduce a Luenberger observer system $\Sigma_{o}$ for $\Sigma$, governed by

$$
\begin{aligned}
\dot{z}(t) & =A z(t)+B u(t)-G\left(y(t)-y_{o}(t)\right), \\
y_{o}(t) & =I z(t) .
\end{aligned}
$$


with $z(0)=0$. Applying the output feedback control

$$
u(t)=F\left(z(t)-x_{\mathrm{ss}}\right)+u_{\mathrm{ss}},
$$

to $\Sigma$, and using the change of coordinates $\xi(t):=x(t)-x_{\mathrm{ss}}$, we get the homogeneous closed loop system

$$
\begin{aligned}
{\left[\begin{array}{c}
\dot{\xi}(t) \\
\dot{e}(t)
\end{array}\right] } & =\left[\begin{array}{cc}
A+B F & -B F \\
0 & A+G C
\end{array}\right]\left[\begin{array}{l}
\xi(t) \\
e(t)
\end{array}\right] \\
\epsilon(t) & =-\left[\begin{array}{cc}
C+D F & -D F
\end{array}\right]\left[\begin{array}{l}
\xi(t) \\
e(t)
\end{array}\right] .
\end{aligned}
$$

where $e(t):=x(t)-z(t)$ is the estimation error, and $\epsilon(t):=$ $r-y(t)$ is the tracking error. The stability of the matrices $A+B F$ and $A+G C$ ensure the estimation and tracking errors vanish and hence asymptotic tracking is achieved. We say that the step response is nonovershooting if $\epsilon(t)$ does not change sign in any component.

In this paper we consider how to design the observer based control law (6) so as to guarantee no overshoot in all components of the output, while also achieving any desired settling time.

\section{Design OF NON-OVERSHOOTING OUTPUT FEEDBACK CONTROLLERS}

Our earlier paper [6] considered the design of nonovershooting state feedback controllers, with control law

$$
u(t)=F\left(x(t)-x_{\mathrm{ss}}\right)+u_{\mathrm{ss}}
$$

In this section, we briefly revise the design procedure for the feedback gain matrix $F$, and then consider the performance of the controller design when employed in conjunction with an observer scheme.

Central to the design method was the choice of a suitable closed loop eigenstructure, consisting of eigenvalues $\mathcal{L}=$ $\left\{\lambda_{1}, \ldots, \lambda_{n}\right\} \in \mathbb{C}$ and eigenvectors $\mathcal{V}=\left\{v_{1}, \ldots, v_{n}\right\} \subset \mathbb{C}^{n}$. The papers [6] offered several design procedures, contingent upon the number of zeros of $\Sigma$ lying in the left hand complex plane (LHP). Theorem 3.1 of [6] assumed that $\Sigma$ has $n-$ $p$ distinct LHP zeros $\left\{z_{1}, \ldots, z_{n-p}\right\}$. Eigenvalues $\mathcal{L}$ were chosen thus: for $i \in\{1, \ldots, n-p\}$, we choose $\lambda_{i}=z_{i}$. For $i \in\{n-p+1, \ldots, n\}$, the remaining closed loop poles $\lambda_{i}$ were freely chosen to be any real distinct stable modes. ${ }^{1}$ To obtain $\mathcal{V}$, we first introduced the set $\mathcal{S}=\left\{s_{1}, \ldots, s_{n}\right\} \subset \mathbb{R}^{p}$ where

$$
s_{i}=\left\{\begin{array}{cl}
0 & \text { for } i \in\{1, \ldots, n-p\} \\
e_{1} & \text { for } i=n-p+1 \\
\vdots & \\
e_{p} & \text { for } i=n .
\end{array}\right.
$$

and $\left\{e_{1}, \ldots, e_{p}\right\}$ is the canonical basis of $\mathbb{R}^{p}$. We then obtained sets $\mathcal{V}=\left\{v_{1}, \ldots, v_{n}\right\} \subset \mathbb{C}^{n}$ and $\mathcal{W}=$ $\left\{w_{1}, \ldots, w_{n}\right\} \subset \mathbb{C}^{p}$ by solving the Rosenbrook matrix equation

$$
\left[\begin{array}{cc}
\lambda_{i} I-A & -B \\
C & D
\end{array}\right]\left[\begin{array}{c}
v_{i} \\
w_{i}
\end{array}\right]=\left[\begin{array}{c}
0 \\
s_{i}
\end{array}\right]
$$

\footnotetext{
${ }^{1}$ If $\Sigma$ has any uncontrollable stable modes, it can be shown that these will also be zeros of $\Sigma$, because $\Sigma$ is invertible. Thus these modes are automatically included in $\mathcal{L}$.
}

for each $s_{i} \in \mathcal{S}$. The sets $\mathcal{L}, \mathcal{V}$ and $\mathcal{W}$ were shown to meet the requirements of Proposition 1 in the classic paper [5] by B.C. Moore, and the procedure given in that paper was then used to obtain a suitable gain matrix $F$ such that $A+B F$ has the desired eigenstructure. The vectors in $\mathcal{V}$ satisfy, for all $i \in\{1, \ldots, n\}$,

$$
\begin{aligned}
& (A+B F) v_{i}=\lambda_{i} v_{i}, \\
& (C+D F) v_{i}=s_{i} .
\end{aligned}
$$

Theorem 3.1 of [6] then showed that with $F$ designed in this way, the state feedback control law (7) would yield an output $y(t)$ for $\Sigma$ that was nonovershooting, from all initial conditions $x_{0}$.

To implement the output feedback control law (6), we first design $F$ according to the above state feedback scheme. Next we must choose some observer pole locations $\left\{\lambda_{n+1}, \ldots, \lambda_{2 n}\right\}$; these may be chosen arbitrarily, provided they all satisfy

$$
\lambda_{i}<\lambda_{n-p+j}
$$

for all $i \in\{n+1, \ldots, 2 n\}$ and all $j \in\{1, \ldots, p\}$. Using the MATLAB command place yields $G$ such that $A+G C$ has the desired observer poles.

To consider the transient performance of the step response arising from the observer scheme (4)-(6), we first introduce matrices

$$
\begin{aligned}
\Lambda & =\left[\begin{array}{cc}
A+B F & -B F \\
0 & A+G C
\end{array}\right] \\
\Gamma & =-\left[\begin{array}{ll}
C+D F & -D F
\end{array}\right] \\
Z(t) & =\left[\begin{array}{l}
\xi(t) \\
e(t)
\end{array}\right]
\end{aligned}
$$

Then $\Lambda$ has eigenvalues $\left\{\lambda_{1}, \ldots, \lambda_{2 n}\right\}$ and eigenvectors $\overline{\mathcal{V}}=$ $\left\{\bar{v}_{1}, \ldots, \bar{v}_{2 n}\right\} \subset \mathbb{C}^{2 n}$. For $i \in\{1, \ldots, n\}$, the eigenvectors $\bar{v}_{i}$ are of the form $\bar{v}_{i}=\left[\begin{array}{ll}v_{i} & 0_{n}\end{array}\right]^{\top}$, where the $v_{i}$ are the eigenvectors in $\mathcal{V}$ and $0_{n}$ is a zero vector of length $n$. For any initial condition $Z(0)=\left[\xi(0)^{\top} e(0)^{\top}\right]^{\top} \in \mathbb{R}^{2 n}$, the tracking error is given by

$$
\epsilon(t)=\Gamma e^{\Lambda t} Z_{0}
$$

As the eigenvectors in $\overline{\mathcal{V}}$ are linearly independent, the matrix $V:=\left[\begin{array}{llll}\bar{v}_{1} & \bar{v}_{2} & \ldots & \bar{v}_{2 n}\end{array}\right]$ is invertible. Introduce $\alpha:=$ $\left[\begin{array}{llll}\alpha_{1} & \alpha_{2} & \ldots & \alpha_{2 n}\end{array}\right]^{\top}=V^{-1} Z_{0}$. The tracking error can be expressed as

$$
\epsilon(t)=\sum_{i=1}^{2 n} \Gamma \bar{v}_{i} \alpha_{i} e^{\lambda_{i} t}
$$

For $i \in\{1, \ldots, n\}$, we have

$$
\begin{aligned}
\Gamma \bar{v}_{i} & =\left[\begin{array}{ll}
C+D F & -D F
\end{array}\right]\left[\begin{array}{ll}
v_{i} & 0_{n}
\end{array}\right]^{\top} \\
& =(C+D F) v_{i} \\
& =s_{i}
\end{aligned}
$$

from (10)-(11). Hence the tracking error can be expressed as

$$
\epsilon(t)=\sum_{i=n-p+1}^{n} e_{i-(n-p)} \alpha_{i} e^{\lambda_{i} t}+\sum_{i=n+1}^{2 n} \Gamma \bar{v}_{i} \alpha_{i} e^{\lambda_{i} t}
$$


If let $\Gamma_{j}$ denote the $j$-th row of $\Gamma$, for $j \in\{1, \ldots, p\}$, then we may express $\epsilon_{j}(t)$, the $j$-th component of $\epsilon(t)$ as

$$
\epsilon_{j}(t)=\alpha_{n-p+j} e^{\lambda_{n-p+j} t}+\sum_{i=n+1}^{2 n} \Gamma_{j} \bar{v}_{i} \alpha_{i} e^{\lambda_{i} t}
$$

Since the observer poles satisfy (12), it follows that a sufficient condition to ensure $\epsilon_{j}(t)$ does not change sign is

$$
\left|\alpha_{n-p+j}\right|>\left|\sum_{i=n+1}^{2 n} \Gamma_{j} \bar{v}_{i} \alpha_{i}\right|
$$

Since $\alpha_{n-p+j}$ depend only upon $\xi(0)=x_{0}-x_{s s}$, while for $i \in\{n+1, \ldots, 2 n\}$ the $\alpha_{i}$ depend upon $e(0)$, it is clear that for any given initial state $x_{0},(21)$ will be satisfied for all $j \in\{1, \ldots, p\}$ if the initial estimation error $e(0)$ is sufficiently small. Summarising the above, we have the following theorem:

Theorem 3.1: Assume that $\Sigma$ satisfies Assumptions 2.1 and has at least $n-p$ stable zeros. Let $F$ and $G$ be defined as above, let $r \in \mathbb{R}^{p}$ be any step reference, and let $x_{0} \in \mathbb{R}^{n}$ be any initial condition. Then applying the output feedback control law $u(t)$ given in (6) to $\Sigma$ yields an output $y(t)$ that will asymptotically track $r$ without overshoot, if the initial error $e(0)$ satisfies (21) for all $j \in\{1, \ldots, p\}$.

Remark 3.1: As the $\lambda_{i}$ for $i \in\{n-p+1, \ldots, n\}$ can be freely chosen to be any distinct real stable modes (provided they are distinct from the stable invariant zeros of $\Sigma$, and provided also that the resulting $\mathcal{V}$ is linearly independent), the rate of convergence of the output trajectory to the target reference can be chosen to be arbitrarily fast. Note also that $F$ is independent of both $r$ and $x_{0}$. Hence, the same $F$ can be used to achieve non-overshooting convergence for any $r \in \mathbb{R}^{p}$ and any $x_{0} \in \mathbb{R}^{n}$. The values of $r$ and $x_{0}$ enter the control law $u$ only through the values of $x_{\mathrm{ss}}$ and $u_{\mathrm{ss}}$.

Remark 3.2: The principal limitation of Theorem 3.1 is that $\Sigma$ is assumed to have $n-p$ stable zeros. Theorem 3.2 of [6] considered the design of a nonovershooting state feedback controller for the cases where $\Sigma$ has $n-2 p$ stable zeros. If the resulting state feedback gain matrix is incorporated into the output feedback law $u(t)$ in (6), then arguments similar to the above will again show that the output achieves nonovershooting reference tracking, provided the initial error estimate is sufficiently small.

Remark 3.3: The bound in (21) is rather conservative. A somewhat less conservative bound may be obtained as follows. For each $j \in\{1, \ldots, p\}$, define $k_{j}$ to be the largest integer such that scalars $\Gamma_{j} \bar{v}_{i} \alpha_{i}$ all have the same sign as $\alpha_{n-p+j}$, for all $i \in\left\{n+1, \ldots, n+j_{k}\right\}$. Now a sufficient condition to ensure $\epsilon_{j}(t)$ does not change sign is

$$
\left|\alpha_{n-p+j}+\sum_{i=n+1}^{n+k_{j}} \Gamma_{j} \bar{v}_{i} \alpha_{i}\right|>\left|\sum_{i=n+k_{j}+1}^{2 n} \Gamma_{j} \bar{v}_{i} \alpha_{i}\right|
$$

Note that $k_{j}$ may equal zero, in which case (21) and (22) coincide.

\section{REDUCED ORDER OBSERVERS}

In this section we consider the implementation of the nonovershooting output feedback controller design in conjunction with a reduced order observer. Applying the change of coordinates described by $T=\left[\begin{array}{ll}T_{1} & T_{2}\end{array}\right]$ with $T_{1}=C^{\dagger}$ and $\operatorname{im} T_{2}=\operatorname{ker} C$, we obtain the equivalent system

$$
\begin{aligned}
A^{\prime} & =T^{-1} A T=\left[\begin{array}{cc}
A_{11}^{\prime} & A_{12}^{\prime} \\
A_{21}^{\prime} & A_{22}^{\prime}
\end{array}\right], \\
B^{\prime} & =T^{-1} B=\left[\begin{array}{c}
B_{1}^{\prime} \\
B_{2}^{\prime}
\end{array}\right], \\
C^{\prime} & =C T=\left[\begin{array}{ll}
I_{p} & 0_{p \times(n-p)}
\end{array}\right], \\
D^{\prime} & =D,
\end{aligned}
$$

where $A^{\prime}$ and $B^{\prime}$ are partitioned conformably with $C^{\prime}$, and the new state $x^{\prime}(t)=T^{-1} x(t)$ is partitioned accordingly. The equations of the system become

$$
\begin{aligned}
{\left[\begin{array}{c}
\dot{x}_{1}^{\prime}(t) \\
\dot{x}_{2}^{\prime}(t)
\end{array}\right] } & =\left[\begin{array}{ll}
A_{11}^{\prime} & A_{12}^{\prime} \\
A_{21}^{\prime} & A_{22}^{\prime}
\end{array}\right]\left[\begin{array}{l}
x_{1}^{\prime}(t) \\
x_{2}^{\prime}(t)
\end{array}\right]+\left[\begin{array}{l}
B_{1}^{\prime} \\
B_{2}^{\prime}
\end{array}\right] u(t) \\
y(t) & =\left[\begin{array}{ll}
I_{p} & 0_{p \times(n-p)}
\end{array}\right]\left[\begin{array}{l}
x_{1}^{\prime}(t) \\
x_{2}^{\prime}(t)
\end{array}\right]+D u(t) .
\end{aligned}
$$

Assumption 2.1 implies $\left(A^{\prime}, B^{\prime}\right)$ is stabilizable and $\left(A_{22}^{\prime}, A_{12}^{\prime}\right)$ is detectable, so we can choose $F$ and $G$ so that $A^{\prime}+B^{\prime} F$ and $\left(A_{22}^{\prime}+G A_{12}^{\prime}\right)$ are both stable matrices, and there exist vectors $x_{\mathrm{ss}} \in \mathbb{R}^{n}$ and $u_{\mathrm{ss}} \in \mathbb{R}^{m}$ exist satisfying

$$
\begin{aligned}
& 0=A^{\prime} x_{\mathrm{ss}}+B^{\prime} u_{\mathrm{ss}} \\
& r=C^{\prime} x_{\mathrm{ss}}+D^{\prime} u_{\mathrm{ss}}
\end{aligned}
$$

for any $r \in \mathbb{R}^{p}$. The state of the reduced order observer is $z(t)=x_{2}^{\prime}(t)+G y_{0}(t)$. By denoting

$$
\begin{aligned}
N & :=A_{22}^{\prime}+G A_{12}^{\prime} \\
M & :=\left(A_{22}^{\prime}+G A_{12}^{\prime}\right) G+A_{21}^{\prime}+G A_{12}^{\prime} \\
L & :=B_{2}^{\prime}+G B_{1}^{\prime},
\end{aligned}
$$

we obtain the reduced order observer system $\Sigma_{\text {red }}$

$$
\dot{z}(t)=N z(t)+M y_{0}(t)+L u(t) .
$$

Applying the reduced order control law

$$
u(t)=F\left(\left[\begin{array}{c}
x_{1}^{\prime}(t) \\
z(t)-G y_{0}(t)
\end{array}\right]-x_{\mathrm{ss}}\right)+u_{\mathrm{ss}}
$$

to $\Sigma$ and introducing the change of coordinates $\xi(t):=$ $x(t)-x_{\mathrm{ss}}$, we get

$$
\begin{aligned}
{\left[\begin{array}{c}
\dot{\xi}(t) \\
\dot{e}(t)
\end{array}\right] } & =\left[\begin{array}{cc}
A^{\prime}+B^{\prime} F & -B^{\prime} F I_{0 n p} \\
0 & N
\end{array}\right]\left[\begin{array}{l}
\xi(t) \\
e(t)
\end{array}\right] \\
\epsilon(t) & =-\left[\begin{array}{cc}
C^{\prime}+D F & -D F I_{0 n p}
\end{array}\right]\left[\begin{array}{l}
\xi(t) \\
e(t)
\end{array}\right]
\end{aligned}
$$

where $e(t):=G x_{1}^{\prime}(t)+x_{2}^{\prime}(t)-z(t)$ is the estimation error, $\epsilon(t):=r-y(t)$ is the tracking error, and we have written $I_{0 n p}:=\left[\begin{array}{c}0 \\ I_{n-p}\end{array}\right]$ for brevity.

The implementation of the reduced order output feedback control law (26) is quite similar to that for the full order observer. We first design $F$ according to the above state 
feedback scheme. Next we must choose some observer pole locations $\left\{\lambda_{n+1}, \ldots, \lambda_{2 n-p}\right\}$; these may be chosen arbitrarily, provided they satisfy

$$
\lambda_{i}<\lambda_{n-p+j}
$$

for all $i \in\{n+1, \ldots, 2 n-p\}$ and all $j \in\{1, \ldots, p\}$.

To consider the transient performance of the step response arising from the observer scheme (25)-(26), we first introduce matrices

$$
\begin{aligned}
& \Lambda=\left[\begin{array}{cc}
A^{\prime}+B^{\prime} F & -B^{\prime} F I_{0 n p} \\
0 & N
\end{array}\right] \\
& \Gamma=-\left[\begin{array}{ll}
C^{\prime}+D F & -D F I_{0 n p}
\end{array}\right] \\
& Z(t)=\left[\begin{array}{l}
\xi(t) \\
e(t)
\end{array}\right]
\end{aligned}
$$

Then $\Lambda$ has eigenvalues $\left\{\lambda_{1}, \ldots, \lambda_{2 n-p}\right\}$ and eigenvectors $\overline{\mathcal{V}}=\left\{\bar{v}_{1}, \ldots, \bar{v}_{2 n-p}\right\} \subset \mathbb{C}^{2 n-p}$. For any initial condition $Z(0)=\left[\xi(0)^{\top} e(0)^{\top}\right]^{\top} \in \mathbb{R}^{2 n-p}$, the tracking error is given by

$$
\epsilon(t)=\Gamma e^{\Lambda t} Z_{0}
$$

Introduce $\alpha:=\left[\begin{array}{llll}\alpha_{1} & \alpha_{2} & \ldots & \alpha_{2 n-p}\end{array}\right]^{\top}=V^{-1} Z_{0}$. Similarly to full order case, it follows that the tracking error can be expressed by

$$
\epsilon(t)=\sum_{i=n-p+1}^{n} e_{i-(n-p)} \alpha_{i} e^{\lambda_{i} t}+\sum_{i=n+1}^{2 n-p} \Gamma \bar{v}_{i} \alpha_{i} e^{\lambda_{i} t}
$$

If we let $\Gamma_{j}$ denote the $j$-th row of $\Gamma$, for $j \in\{1, \ldots, p\}$, then we may express $\epsilon_{j}(t)$, the $j$-th component of $\epsilon(t)$ as

$$
\epsilon_{j}(t)=\alpha_{n-p+j} e^{\lambda_{n-p+j} t}+\sum_{i=n+1}^{2 n-p} \Gamma_{j} \bar{v}_{i} \alpha_{i} e^{\lambda_{i} t}
$$

Since the observer poles satisfy $(27), \epsilon_{j}(t)$ will not change sign if

$$
\left|\alpha_{n-p+j}\right|>\left|\sum_{i=n+1}^{2 n-p} \Gamma_{j} \bar{v}_{i} \alpha_{i}\right|
$$

It is again clear that for any given initial state $x_{0},(31)$ will be satisfied for all $j \in\{1, \ldots, p\}$ if the initial estimation error $e(0)$ is sufficiently small. Summarising the above, we have the following:

Theorem 4.1: Assume that $\Sigma$ satisfies Assumptions 2.1 and has at least $n-p$ stable zeros. Let $F$ and $G$ be defined as above, let $r \in \mathbb{R}^{p}$ be any step reference, and let $x_{0} \in \mathbb{R}^{n}$ be any initial condition. Then applying the reduced order output feedback control law $u(t)$ given in (26) to $\Sigma$ yields an output $y(t)$ that will asymptotically track $r$ without overshoot, if the initial error $e(0)$ satisfies (31) for all $j \in\{1, \ldots, p\}$.

Remark 4.1: As for the full order case, it is similarly possible to take the feedback gain matrix $F$ obtained from Theorem 3.2 of [6], where $\Sigma$ has $n-2 p$ stable zeros, and incorporate it into the reduced order output feedback law $u(t)$ in (26). The output also achieves nonovershooting reference tracking, provided the initial error estimate is sufficiently small. Likewise, the bound in (31) may be replaced with a less conservative one similar to (22).

\section{EXAMPLE}

Example 5.1: Consider the MIMO system $\Sigma_{1}=$ $(A, B, C, D)$ with

$$
\begin{array}{cc}
A=\left[\begin{array}{cccc}
0 & 0 & -4 & 6 \\
0 & -4 & 0 & 0 \\
-7 & 2 & 7 & 2 \\
5 & 0 & -4 & 0
\end{array}\right] \quad B=\left[\begin{array}{cc}
0 & 3 \\
-1 & 10 \\
0 & 0 \\
0 & 0
\end{array}\right] \\
C=\left[\begin{array}{cccc}
0 & 2 & 9 & 7 \\
-1 & 0 & -10 & 1
\end{array}\right] \quad D=\left[\begin{array}{cc}
-2 & 0 \\
0 & 5
\end{array}\right] .
\end{array}
$$

Hence, $\Sigma_{1}$ is an invertible system with $n=4, p=2$ and $m=2$. The system is open-loop unstable with poles at $\{10.8977,1.4161,-4,-5.3137\}$. Also, the system is of non-minimum phase with invariant zeros at $\{-5.2402 \pm$ $1.8728 i, 9.0805,4\}$. Thus $\Sigma_{1}$ satisfies Assumption 2.1 and has $n-p=2$ stable zeros. Let assume the desired step reference is $r=\left[\begin{array}{ll}1 & -1\end{array}\right]^{\top}$. Applying the state feedback design method from [6] yields feedback gain matrix

$$
F=\left[\begin{array}{cccc}
-53.2632 & 11.6840 & 74.8301 & -7.3138 \\
-2.5894 & 0.0972 & 3.0241 & -2.5160
\end{array}\right] \text {. }
$$

with closed loop poles at $\mathcal{L}=\{-5.2402 \pm$ $1.8728 i,-3,-2\}$. Here the complex poles were chosen to coincide with the stable zeros of $\Sigma_{1}$, the other two poles were chosen arbitrarily. We also choose observer poles at $\{-11,-10,-9,-8\}$ to satisfy (12). For an initial condition of $x_{0}=0$, we obtain $\xi(0)=-[0.0597,-0.1291,0.0747,0.0768]^{\top}$. Let us assume an initial estimator error of $e(0)=\delta \xi(0)$, where $\delta$ is a variable scalar. Applying the observer feedback $u(t)$ in (6) to $\Sigma_{1}$ where $\delta=0$ yields the nonovershooting output shown in Figure 1. This corresponds to the case where state feedback is used.

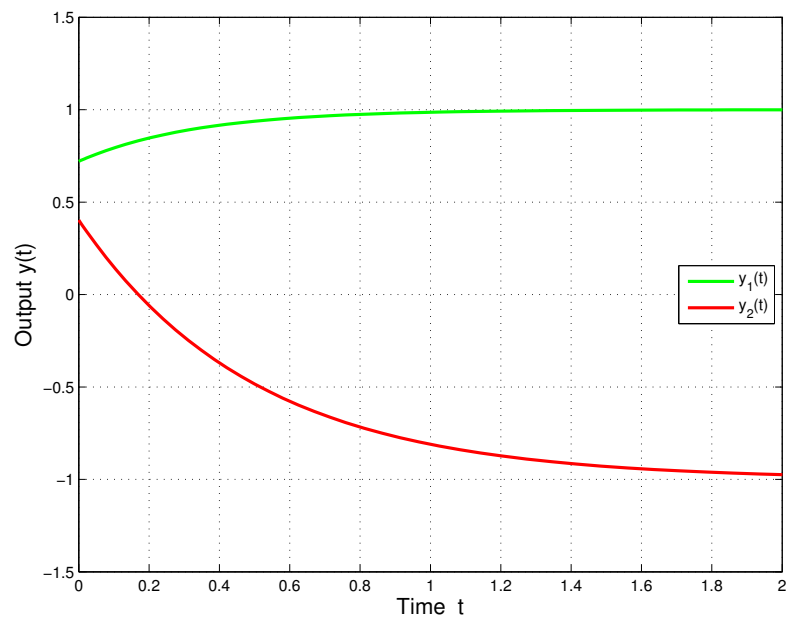

Fig. 1. $\Sigma_{1}$ step response using state feedback

Applying the full order observer feedback $u(t)$ in (6) to $\Sigma_{1}$ where $\delta=1.2 \%$ yields the nonovershooting output shown in Figure 2. We note that increasing $\delta$ to $1.3 \%$ does yield overshoot. 


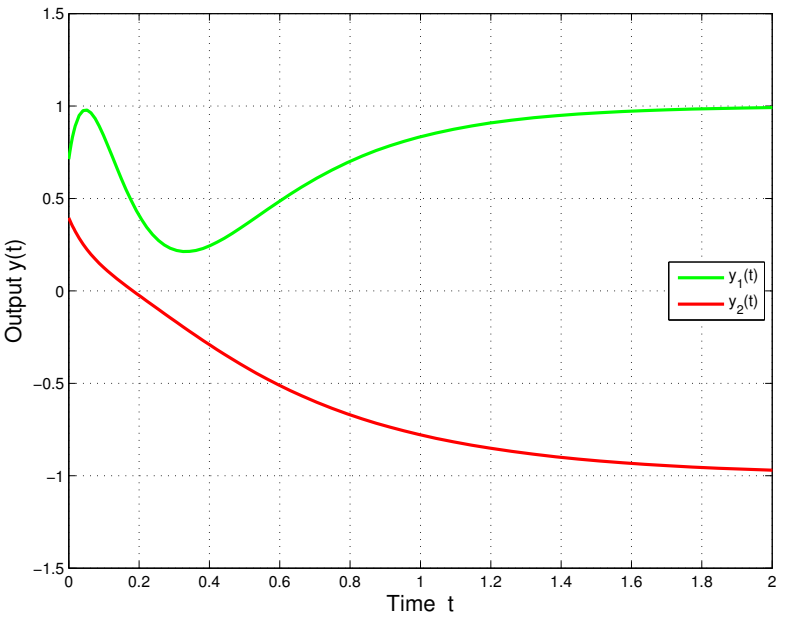

Fig. 2. $\Sigma_{1}$ step response using full order observer

\section{REFERENCES}

[1] M. Bement and S. Jayasuriya, "Use of state feedback to achieve a nonovershooting step response for a class of nonminimum phase systems", Journal of Dynamical Systems, Measurement and Control, vol. 126, pp. 657-660, 2004.

[2] M. Bement and S. Jayasuriya, "Construction of a set of nonovershooting Tracking controllers", Journal of Dynamical Systems, Measurement and Control, vol. 126, pp. 558-567, 2004.

[3] S. Darbha and S.P. Bhattacharyya, "On the synthesis of controllers for a nonovershooting step response", IEEE Transactions on Automatic Control, vol. 48, pp. 797-799, 2003.

[4] Y. He, B.M. Chen, and C. Wu, "Composite nonlinear control with state and measurement feedback for general multivariable systems with input saturation", Systems \& Control Letters, vol. 54, pp. 455469, 2005.

[5] B.C. Moore, "On the Flexibility Offered by State Feedback in Multivariable systems Beyond Closed Loop Eigenvalue Assignment", IEEE Transactions on Automatic Control, pp. 689-692, 1976.

[6] R. Schmid and L. Ntogramatzidis, "Nonovershooting linear multivariable state feedback tracking controllers", Proceedings 17th IFAC World Congress, Seoul, 2008.
To obtain a reduced order observer, we again choose open loop poles for $A^{\prime}+B^{\prime} F$ at $\{-5.2402 \pm 1.8728 i,-3,-2\}$, and choose estimator poles at $\left\{\begin{array}{ll}-9, & -8\}\end{array}\right\}$ to satisfy (27). For the initial condition $x_{0}=0$, we obtain $\xi(0)=-[0.0054,-0.0028,-0.1099,0.1118]^{\top}$. Let us assume an initial estimator error of $e(0)=\delta \bar{\xi}(0)$, where $\delta$ is a variable scalar and $\bar{\xi}$ indicates the third and fourth components of $\xi$. Applying the reduced order observer feedback $u(t)$ in (26) to $\Sigma_{1}$ where $\delta=2.7 \%$ yields the nonovershooting output shown in Figure 3. We note that increasing $\delta$ to $2.8 \%$ does yield overshoot.

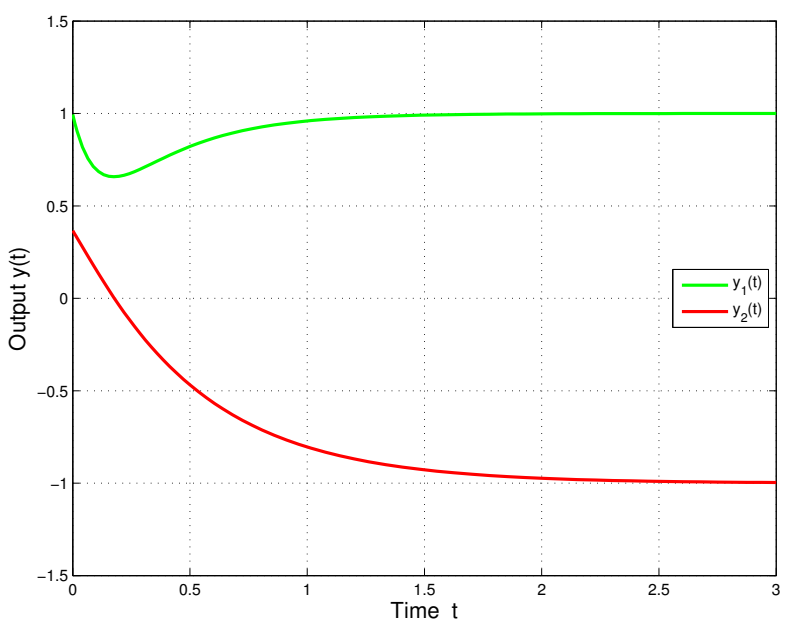

Fig. 3. $\Sigma_{1}$ step response using reduced order observer

\section{CONCLUSION}

We have considered the use of dynamic output feedback to obtain a nonovershooting step response, for MIMO LTI systems. It was shown that both the full order and reduced order Luenberger observers can achieve a nonovershooting step response if the error of the initial state estimate is sufficiently small. This enables the state feedback scheme introduced in [6] to be more practically implemented. 